\section{Interactions between Glucagon and Other Counterregulatory Hormones during Normoglycemic and Hypoglycemic Exercise in Dogs}

\author{
David H. Wasserman, H. Lavina A. Lickley, \\ and Mladen Vranic \\ Departments of Physiology, Medicine, and Surgery, University of \\ Toronto, Toronto, Ontario, Canada M5S $1 A 8$
}

only $3.7 \pm 0.8 \mathrm{mg} / \mathrm{kg}-\mathrm{min}$. During glucose replacement, IRG decreased by $109 \pm 30 \mathrm{pg} / \mathrm{ml}$ and the IRI response did not differ from the response to normal exercise. $\mathbf{R}_{a}$ rose minimally by $1.5 \pm 0.3 \mathrm{mg} / \mathrm{kg}$-min. The changes in $E, C, R_{d}$, and $L$ were restored to normal, whereas the FFA response remained excessive. In all protocols increments in $R_{a}$ were directly correlated to the IRG/IRI molar ratio while no correlation could be demonstrated between epinephrine or norepinephrine and $\mathbf{R}_{\mathrm{a}}$. In conclusion, (a) glucagon controlled $\sim 70 \%$ of the increase of $R_{a}$ during exercise. This became evident when counterregulatory responses to hypoglycemia (E and $C$ ) were obviated by glucose replacement; $(b)$ increments in $\mathbf{R}_{\mathrm{a}}$ were strongly correlated to the IRG/IRI molar ratio but not to the plasma catecholamine concentration; $(c)$ the main role of $\mathrm{E}$ in hypoglycemia was to limit glucose uptake by the muscle; $(d)$ with glucagon suppression, glucose production was deficient but a further decline of glucose was prevented through the peripheral effects of $\mathrm{E} ;(e)$ the hypoglycemic stimulus for $\mathrm{E}$ secretion was facilitated by exercise; and $(f)$ we hypothesize that an important role of glucagon during exercise could be to spare muscle glycogen by stimulating glucose production by the liver.

\section{Introduction}

During exercise of moderate intensity, glucose uptake increases to a steady state approximately threefold above resting values (1-3), yet plasma glucose usually remains constant. To maintain euglycemia, hepatic glucose production $\left(R_{a}\right)^{1}$ must increase to match peripheral glucose uptake. Increased sympathetic drive (4), an increase in glucagon $(5,6)$ and circulating catecholamines $(6,7)$, and a decrease in insulin $(2,7)$ are all characteristic

1. Abbreviations used in this paper: $\mathrm{G}$, glucose; IRG, immunoreactive glucagon; IRI, immunoreactive insulin; $\mathbf{R}_{\mathbf{a}}$, glucose production; $\mathbf{R}_{\mathbf{d}}$, glucose utilization; ST, somatostatin. 
responses to exercise. All of these hormonal and neural changes are known to increase $R_{a}(8)$.

In earlier work, using somatostatin to suppress glucagon during exercise, we showed that glucagon is an important regulator of $\mathbf{R}_{\mathrm{a}}$ during both steady and nonsteady state exercise (9). Glucagon suppression caused a drop in $R_{a}$, resulting in mild hypoglycemia. When glucagon was replaced during somatostatin infusion, normal glucose fluxes were restored. This demonstrated that the fall in $\mathbf{R}_{\mathbf{a}}$ occurred as a result of glucagon suppression and was not due to a direct effect of somatostatin. However, it is possible that hypoglycemia-induced counterregulatory responses may have masked some of the effects of glucagon lack. Indeed, in resting dogs, selective glucagon suppression induced only a small decrease in $R_{a}$, but this decrease was much more pronounced when a euglycemic glucose clamp was established (10). Therefore, in the present study, to quantify the responses of the main counterregulatory hormones to exercise, we measured epinephrine, norepinephrine, and cortisol in addition to immunoreactive glucagon and insulin (IRG and IRI, respectively). Five running dogs served as controls while another five dogs were run with a concurrent infusion of somatostatin, with or without a euglycemic glucose clamp. The objectives of the study were: $(a)$ to establish the role of glucagon in regulating $\mathbf{R}_{\mathrm{a}}$ during exercise when hypoglycemia-induced counterregulation was prevented, $(b)$ to determine whether or not moderate hypoglycemia serves as a stimulus to exaggerated release of the catecholamines and/or cortisol, $(c)$ to delineate the metabolic consequences of any adrenergic counterregulation that may occur, and $(d)$ not only to further delineate the role of glucagon during exercise, but also to gain a better understanding of the interactions between glucagon and the catecholamines in the regulation of glucose fluxes during exercise.

\section{Methods}

Experimental animals. 10 mongrel dogs (both sexes, body weight $17-$ $27 \mathrm{~kg}$ ) were maintained on a high protein diet that consisted of $200 \mathrm{~g}$ dog chow (Ralston Purina Canada Ltd., Mississauga, Ontario) and 400 $g$ beef chunks (Dr. Ballard's, Toronto, Ontario) each day. Dogs were trained to run on a treadmill for at least 2 wk. $4 \mathrm{~d}$ before the first experiment, surgery was performed under general anaesthesia (halothane, nitrous oxide). Three tygon catheters (0.04-inch internal diameter) were placed in the superior vena cava via a jugular vein, for the infusion of tracer, somatostatin (ST), and glucose (G). A tygon catheter (0.05-inch internal diameter) was inserted into a carotid artery for sampling purposes.

Experimental design. Food was withdrawn from the animals $14 \mathrm{~h}$ before each experiment. A primed-infusion of tracer $\left[3-{ }^{3} \mathrm{H}\right]$ glucose was started at time $0800 \mathrm{~h}$. This was followed by a 100-min equilibration period, $40 \mathrm{~min}$ of basal sampling, $90 \mathrm{~min}$ of exercise $(100 \mathrm{~m} / \mathrm{min}$, $12^{\circ}$ slope) and $60 \mathrm{~min}$ of recovery from exercise. Three sets of experimental studies were carried out. (a) Five dogs were studied during exercise alone (control studies); (b) five additional dogs received an infusion of somatostatin (ST, $0.5 \mu \mathrm{g} / \mathrm{kg}$-min) during both the exercise and recovery periods; $(c)$ the same five animals used in $b$ above were also studied on a separate occasion when they received both somatostatin and a constant infusion of glucose (G, $3.5 \mathrm{mg} / \mathrm{kg}$ $\min )$. Studies $b$ and $c$ were carried out in randomized order separated by at least $3 \mathrm{~d}$.

It is possible that a wide range of glucose infusion rates could have maintained euglycemia under the conditions of our study. It was essential, therefore, for the interpretation of our results that euglycemia be maintained without distorting glucose turnover relative to normal exercising values. Fig. 1 compares the total appearance of glucose (exogenous and endogenous sources) into the body pool while Fig. 2 $C$ compares glucose utilization $\left(\mathrm{R}_{\mathrm{d}}\right)$ in exercise alone and exercise plus ST with or without a euglycemic glucose clamp. The glucose infusion rate given during the infusion of glucose plus ST did not distort glucose fluxes when compared with normal exercise values.

Tracer methods. In all experiments, $5 \mu \mathrm{Ci} / \mathrm{ml}$ of $\left[3-{ }^{3} \mathrm{H}\right]$ glucose (New England Nuclear, Lachine, Quebec) was infused at a constant rate $(0.094 \mathrm{ml} / \mathrm{min})$. A priming dose, equivalent to the amount infused in $140 \mathrm{~min}$ was given at the beginning of each experiment. The method of measurement of $R_{\mathbf{a}}$ and $\mathbf{R}_{\mathbf{d}}$ for endogenous glucose has been described previously (11) and extensively validated $(12,13)$.

Laboratory methods. Blood samples for the measurement of labeled and unlabeled glucose, lactate, IRI and cortisol were collected in dried heparinized tubes with sodium fluoride as preservative. Plasma for tracer glucose determination was deproteinized by equal volumes of 5\% zinc sulfate and $0.3 \mathrm{~N}$ barium hydroxide. Deproteinized samples were evaporated to remove tritiated water and then redissolved in distilled water. Samples were counted in Aquasol 11 (New England Nuclear) by liquid scintillation spectrometry. For each experiment, an

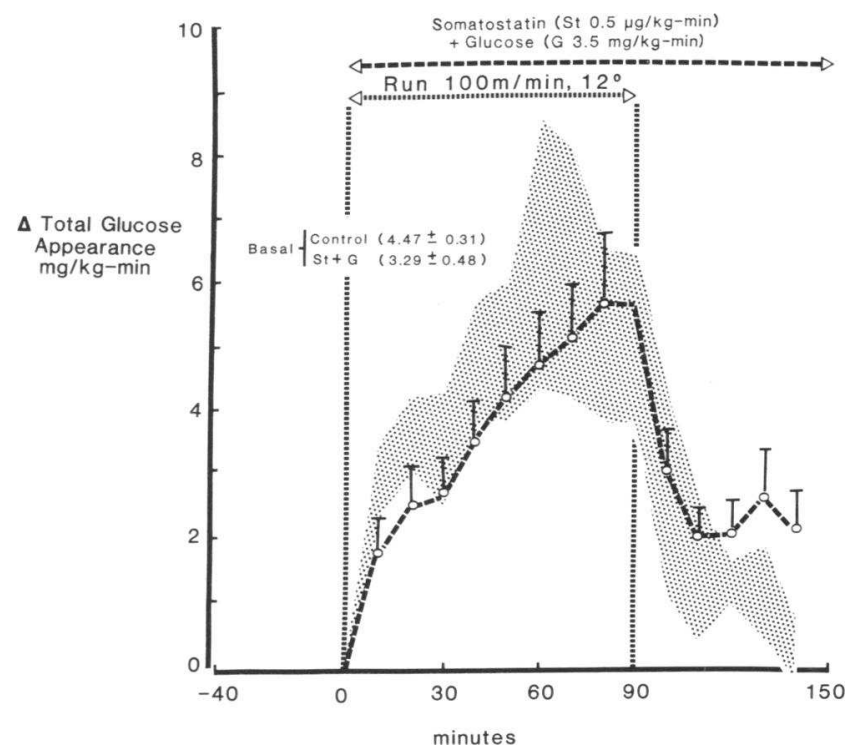

Figure 1. Effect of exercise alone (control, dotted area) or exercise plus somatostatin plus glucose ( $\mathrm{ST}+\mathrm{G},---)$ on the changes in the rate of total glucose appearance. Data are shown as deviations $(\Delta)$ from the basal values. Exercise and somatostatin and glucose infusions were begun at $t=0$. The stippled area represents mean $\pm \mathrm{SE}$ for exercise alone. Vertical bars represent the SEM. $n=5$ for all protocols. 


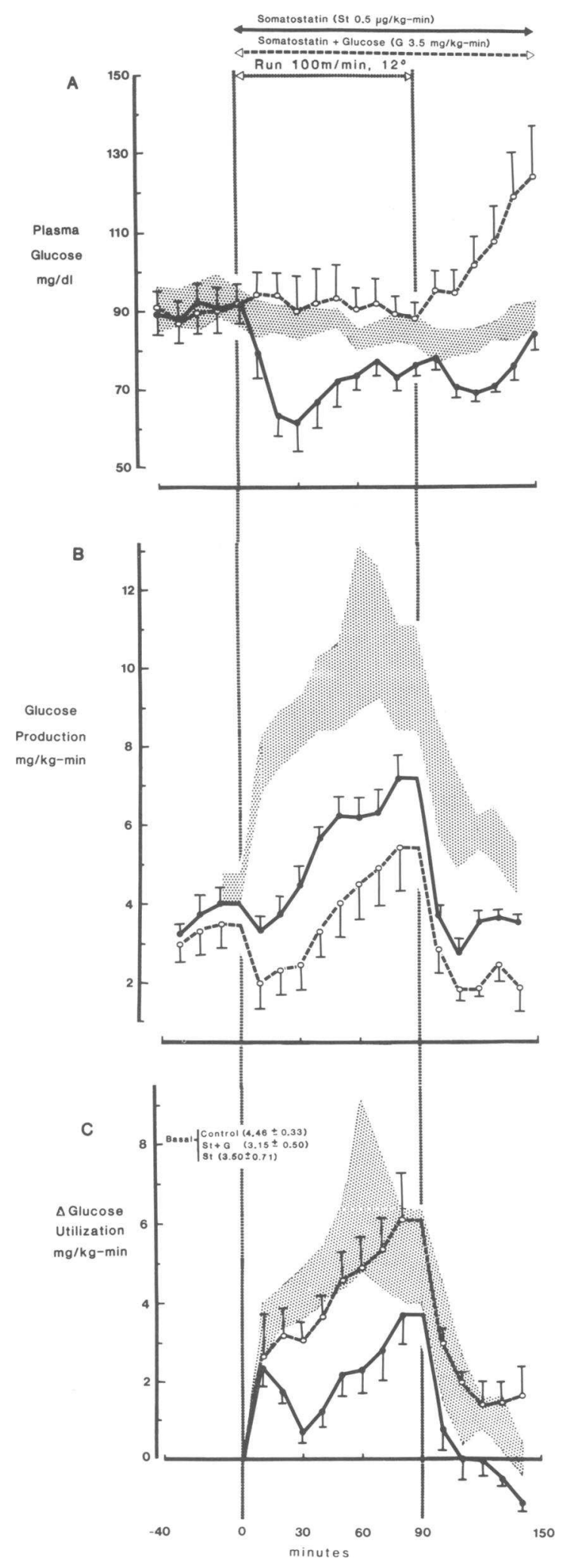

aliquot of the infused tracer solution was prepared as a standard. This was treated in the same way as the plasma samples, and then counted. Plasma glucose concentrations were determined by the glucose oxidase method (glucose Autoanalyzer, Beckman Instruments, Inc., Fullerton, CA). Plasma IRI was assayed by the method of Herbert et al. (14). Cortisol was measured using a modification of a competitive protein binding assay (15). Plasma lactate was analyzed by measuring the enzymatic reduction of nicotine adenine dinucleotide spectrophotometrically (16).

Blood samples for determination of IRG and plasma FFA were collected in $0.1 \mathrm{ml}$ trasylol (2,000 kallikrien IU, FBA Pharmaceuticals, New York) and $0.1 \mathrm{ml}$ EDTA $(24 \mathrm{mg} / \mathrm{ml})$. Plasma glucagon was determined by radioimmunoassay (17) using 30-K antiserum (obtained from R. Unger, Dallas, TX) and $\mathrm{I}^{125}$-glucagon (obtained from Novo Research Institute, Copenhagen, Denmark). Plasma FFA levels were determined by the method described by Ho (18). For the determination of norepinephrine, epinephrine, and dopamine, blood samples were collected in polyethylene tubes containing $2.5 \mathrm{mg}$ glutathione. To each sample, $10 \mu$ lof ethylene glycol-bis-(B-aminoethylether)- $N, N^{1}$-tetraacetic acid (EGTA) was added as anticoagulant. Within $2 \mathrm{~h}$ after sampling the tubes were centrifuged and the plasma was deproteinized with $2 \mathrm{~N}$ $\mathrm{HCLO}_{4}$ and stored at $-70^{\circ} \mathrm{C}$. Analysis was by a radioenzymatic assay (19).

The data in the text and figures are expressed as means \pm SE. Statistical significance was assessed using the unpaired $t$ test or paired $t$ test where applicable. Correlations were examined using Pearson's Correlation Coefficient. Significance was presumed at $\boldsymbol{P}<0.05$.

\section{Results}

Plasma glucose concentrations and glucose turnover. Fig. $2 \mathrm{~A}$ illustrates the effect of the three protocols on plasma glucose concentration. In the control experiments, plasma glucose concentration remained constant throughout exercise. Somatostatin infusion during exercise resulted in hypoglycemia. Plasma glucose fell transiently with a nadir at $30 \mathrm{~min}$ of $62 \pm 6$ $\mathrm{mg} / \mathrm{dl}$. Glucose values were significantly lower from 10 to $\mathbf{4 0}$ $\min (P<0.05)$ after the onset of exercise plus ST than those seen with exercise alone. Glucose replacement during somatostatin infusion restored the plasma glucose concentration to normal levels during exercise. However, after exercise when muscular glucose utilization decreased, hyperglycemia was noted.

Kinetic analysis demonstrated that the hypoglycemia seen when ST was infused during exercise was due to a significantly smaller increase in endogenous glucose production than that seen during exercise alone (Fig. $2 B$ ). With normal exercise $\mathbf{R}_{a}$ rose to a peak of $11.06 \pm 2.15 \mathrm{mg} / \mathrm{kg}$-min after $60 \mathrm{~min}$ of

Figure 2. Effect of exercise alone (control, dotted area), exercise plus somatostatin (ST, $\longrightarrow$ ), or exercise plus somatostatin plus glucose $\left(\mathrm{ST}+\mathrm{G},-\mathrm{-}^{--)}\right.$on $(A)$ plasma glucose concentration $(B)$ the rate of hepatic glucose production, and $(C)$ changes in the rate of glucose utilization. Glucose utilization is shown as deviations $(\Delta)$ from the basal values. Exercise and somatostatin and glucose infusions were begun at $t=0$. The stippled area represents mean $\pm \mathrm{SE}$ for exercise alone. Vertical bars represent the SEM. $n=5$ for all protocols. 
exercise. $R_{\mathrm{a}}$ rose to only $6.21 \pm 0.51 \mathrm{mg} / \mathrm{kg}-\mathrm{min}$ after the same duration with $\mathrm{ST}$-induced glucagon suppression during exercise. The $\mathbf{R}_{\mathbf{a}}$ values in the two studies were significantly different from 10 to $70 \mathrm{~min}(p<0.05$ to 0.005$)$ after the start of exercise. This occurred because the increase in $R_{a}$ was delayed for $\mathbf{4 0} \mathrm{min}$ in the second study. In contrast, $R_{\mathrm{a}}$ rose immediately after the onset of exercise in controls. When glucose was infused with somatostatin the $\mathbf{R}_{\mathrm{a}}$ response was further suppressed and an increase over basal was observed only after 60 min. $\mathbf{R}_{\mathbf{a}}$ was significantly lower with the infusion of ST plus $G$ than during exercise alone for the entire exercise period ( $P$ $<0.05$ to 0.005 ). $R_{a}$ was also significantly lower with the infusion of ST plus $G$ infusion than during infusion of ST alone. In the two normoglycemic protocols $R_{d}$ rose similarly with exercise, reaching a peak increment of $6.19 \pm 1.50 \mathrm{mg} / \mathrm{kg}$ min (Fig. 2 C). With hypoglycemia the increase in $R_{d}$ with exercise was partially suppressed as a peak increase of only $3.69 \pm 0.75 \mathrm{mg} / \mathrm{kg}$-min was attained. $R_{d}$ was significantly lower with hypoglycemia compared with normoglycemic exercise from 20 to $60 \mathrm{~min}(P<0.05$ to 0.005$)$.

Pancreatic hormone responses. Fig. $3 \mathrm{~A}$ shows the responses of IRG to the three exercise studies. During the infusion of ST or ST plus $G$ during exercise, IRG was suppressed below basal levels and far below the response seen with exercise alone, throughout the exercise period $(P<0.025$ to 0.001$)$. The IRG levels were similar with the infusion of ST or ST plus G. The IRG nadir was noted by $30 \mathrm{~min}$ and then increased, approaching basal levels by the end of exercise. As expected, IRI decreased with exercise (Fig. $3 \mathrm{~B}$ ). Levels fell by $10.2 \pm 4.0 \mu \mathrm{U} / \mathrm{ml}$ during exercise alone. There were no significant differences in IRI values in any of the three studies.

When the results of the three protocols were grouped together $R_{a}$ was found to be significantly correlated with IRG ( $r=0.61, P<0.0001)$ as well as to the molar ratio of IRG to IRI $(r=0.76, P<0.0001$, Fig. 4). When the three protocols were analyzed independently IRG/IRI was still significantly correlated with $R_{a}$ in all three studies, but IRG was correlated with $\mathbf{R}_{\mathrm{a}}$ only in the control group.

Adrenal hormone responses. The change in plasma cortisol during the three exercise studies is shown in Fig. $5 \mathrm{~A}$. Cortisol increased only slightly with exercise alone $(2.1 \pm 1.0 \mu \mathrm{g} / \mathrm{dl}$, by $90 \mathrm{~min}$ ). When ST was infused, the increase of cortisol coincided with the fall in plasma glucose. A peak increase of $5.4 \pm 1.5 \mu \mathrm{g} / 100 \mathrm{dl}$ was reached after $50 \mathrm{~min}$ of exercise. The normal cortisol response to exercise was restored when hypoglycemia was prevented. Cortisol values were significantly higher with exercise plus ST than with normoglycemic exercise from 50 to $70 \mathrm{~min}(P<0.05$ to 0.001$)$.

Fig. $5 B$ shows that without hypoglycemia, epinephrine rose gradually to about threefold after $90 \mathrm{~min}$. With the hypoglycemic nadir when glucagon was suppressed during exercise, the increase in epinephrine was accelerated, rising sixfold after only $30 \mathrm{~min}$. Statistical significance between normoglycemic and hypoglycemic exercise was found from 10

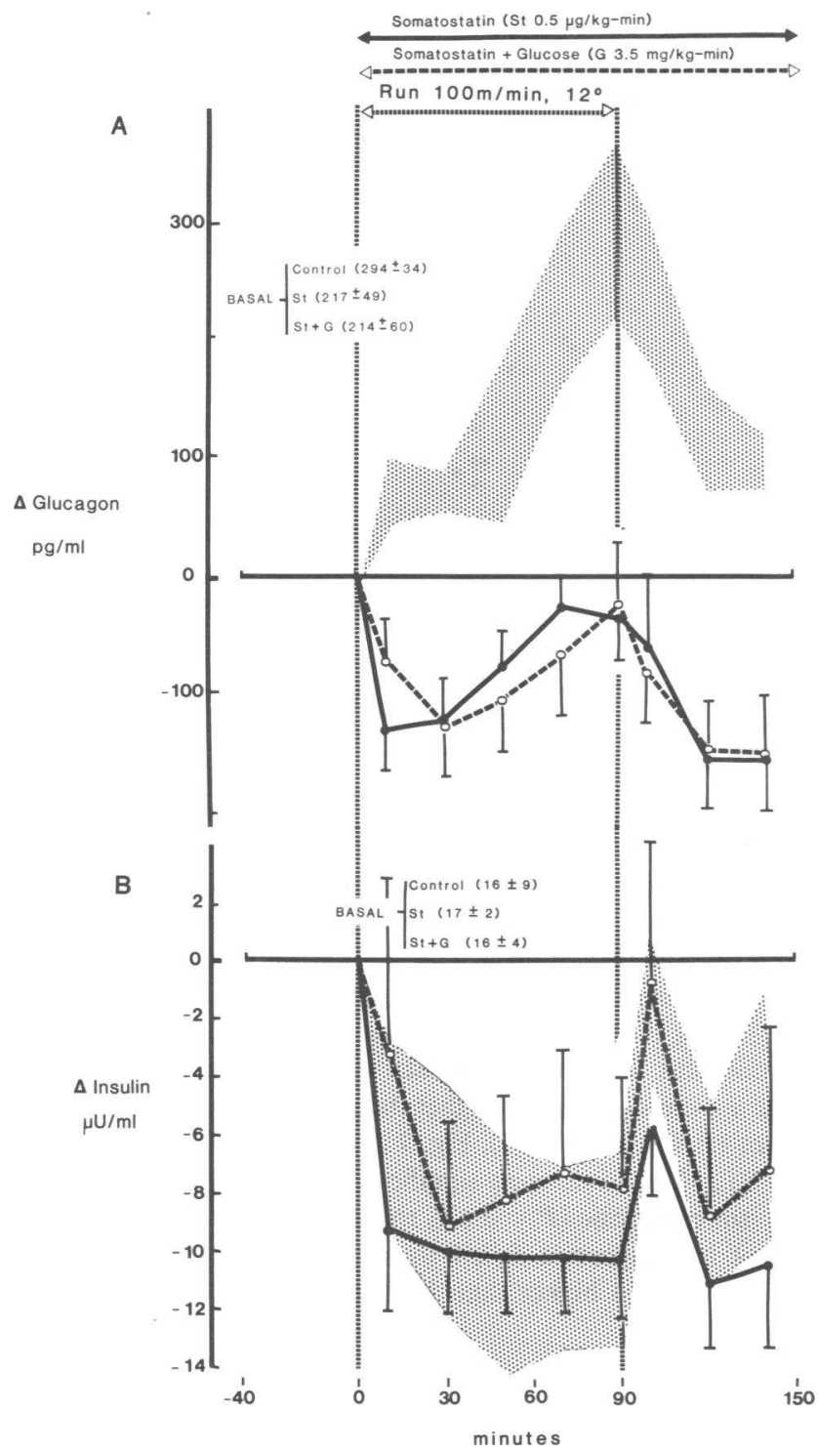

Figure 3. Effect of exercise alone (control, dotted area), exercise plus somatostatin (ST, - - ), or exercise plus somatostatin plus glucose $\left(\mathrm{ST}+\mathrm{G}, \mathrm{-}^{---)}\right.$on $(A)$ changes in glucagon and $(B)$ changes in insulin. Data are shown as deviations $(\Delta)$ from the basal values. Exercise and somatostatin and glucose infusions were begun at $t=0$. The stippled area represents mean \pm SE for exercise alone. Vertical bars represent the SEM. $n=5$ for all protocols.

to $50 \mathrm{~min}(P<0.05$ to 0.005$)$. Norepinephrine increased fourfold and this was not affected by hypoglycemia (Fig. $5 C$ ). Individual epinephrine data during rest and exercise were plotted against the plasma glucose values (Fig. 6). It can be seen that the epinephrine response to exercise was enhanced with a very modest decrease in plasma glucose. Very high 


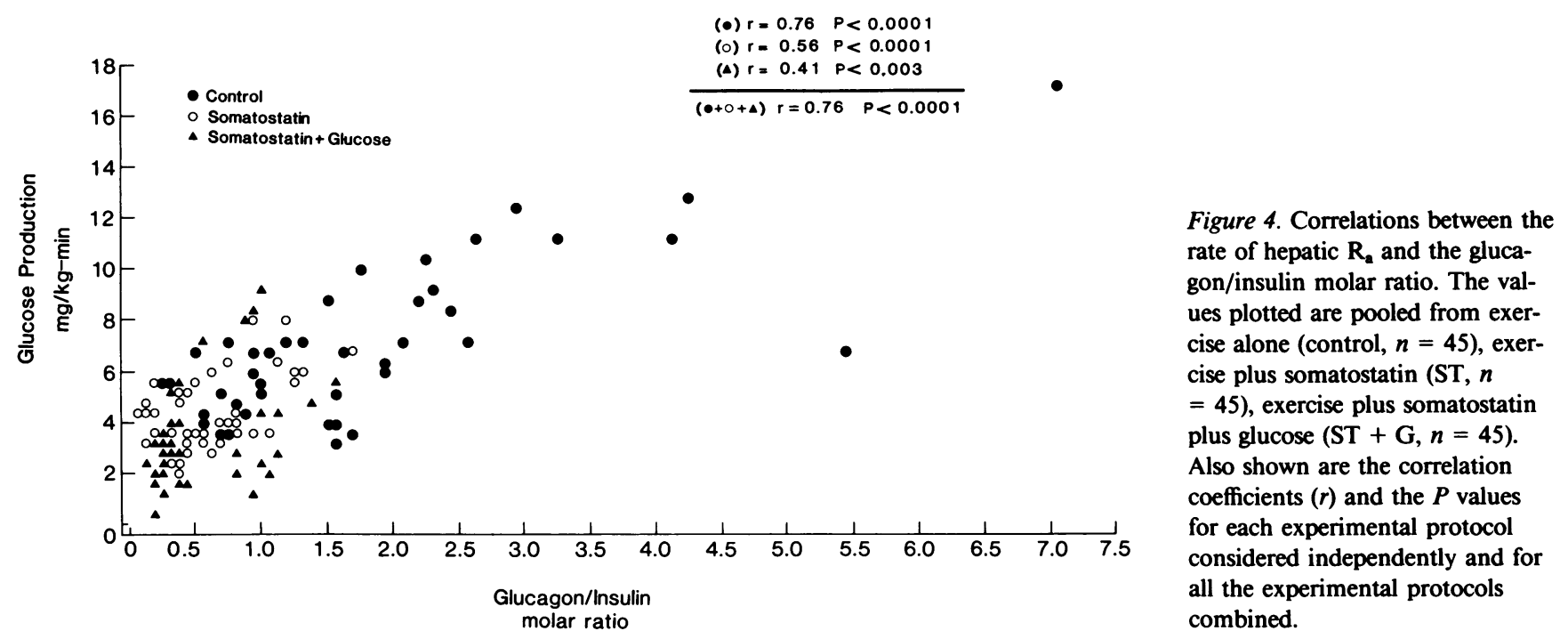

epinephrine values were reached at glucose levels $<85 \mathrm{mg} / \mathrm{dl}$. $R_{a}$ was not significantly correlated to either epinephrine or norepinephrine under any of the three exercise conditions.

Metabolites. The lactate levels during the three exercise protocols are shown in Fig. $7 \mathrm{~A}$. During exercise plus ST, lactate increased by $15.5 \pm 3.8 \mathrm{mg} / \mathrm{dl}$ at $30 \mathrm{~min}$ but by only $6.8 \pm 1.9 \mathrm{mg} / \mathrm{dl}$ during exercise alone and $6.6 \pm 1.9 \mathrm{mg} / \mathrm{dl}$ during exercise plus ST plus G. Lactate values during hypoglycemic exercise were significantly higher than in the two normoglycemic groups $(P<0.05$ to 0.01$)$. Fig. $7 B$ shows that during exercise plus ST, FFA levels were significantly higher than those seen with exercise alone $(P<0.025$ to 0.005$)$. Surprisingly, prevention of hypoglycemia failed to correct this increase as FFA values remained higher than in the control studies $(P<0.05$

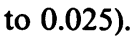

\section{Discussion}

This study not only illustrates the important role of glucagon in exercise but also delineates the nature of the counterregulatory responses that are triggered when glucagon is suppressed. In a previous study we showed that suppression of glucagon, either at the onset of exercise or during steady state exercise, resulted in suppression of $R_{a}$ and transient hypoglycemia (9). However, the role and nature of counterregulation during hypoglycemia was not examined. Also, the full metabolic impact of glucagon suppression could only be revealed if counterregulatory responses were prevented along with glucagon suppression (10). In the resting dog, isolated glucagon deficiency resulted in approximately a $1.5-3.0 \mathrm{mg} / \mathrm{kg}$-min decrement in $\mathbf{R}_{\mathrm{a}}$ when glucose was clamped (10). In our studies, glucagon suppression lowered $R_{a} 4.8 \mathrm{mg} / \mathrm{kg}$-min, suggesting that the exercise-induced increment in $\mathbf{R}_{\mathbf{a}}$ had been affected as well as base-line $\mathbf{R}_{\mathrm{a}}$. However, since insulin is reduced with exercise, hepatic sensitivity to glucagon will probably be increased (2022 ). Thus, although we have clearly shown that the presence of glucagon is important we cannot define quantitatively the relative importance of basal vs. the extra glucagon released during exercise.

To accurately assess the effects of glucagon suppression during exercise in these studies requires that somatostatin does not have an appreciable direct effect on glucose fluxes. In earlier work, we showed (9) that when endogenous glucagon release was suppressed with somatostatin, glucagon replacement ( 4 or $6 \mathrm{ng} / \mathrm{kg}$-min) normalized plasma glucose levels, glucose production, and uptake. Thus, during somatostatin infusion, glucose replacement in this paper and glucagon replacement in our previous work yielded the same results with respect to total glucose appearance and glucose uptake. In the present work, despite receiving exactly the same somatostatin infusion, excess release of epinephrine and cortisol were only seen in the group allowed to become hypoglycemic. Therefore, it is unlikely that any of the changes we observed are a direct result of somatostatin, but instead, a manifestation of glucagon suppression.

In man, acute suppression of glucagon by somatostatin also resulted in hypoglycemia during exercise (23). However, when ST was infused $2 \mathrm{~h}$ before and during exercise, the increase in splanchnic glucose output was not attenuated. This could be explained by the fact that glucagon levels were still substantial, and the liver had been exposed to low IRI levels for a prolonged period of time. These observations in man were similar to our observations in depancreatized dogs in which the mere presence of glucagon was sufficient to control hepatic glucose production during exercise (2). It should be noted that there is a difference in the glucagon response to 


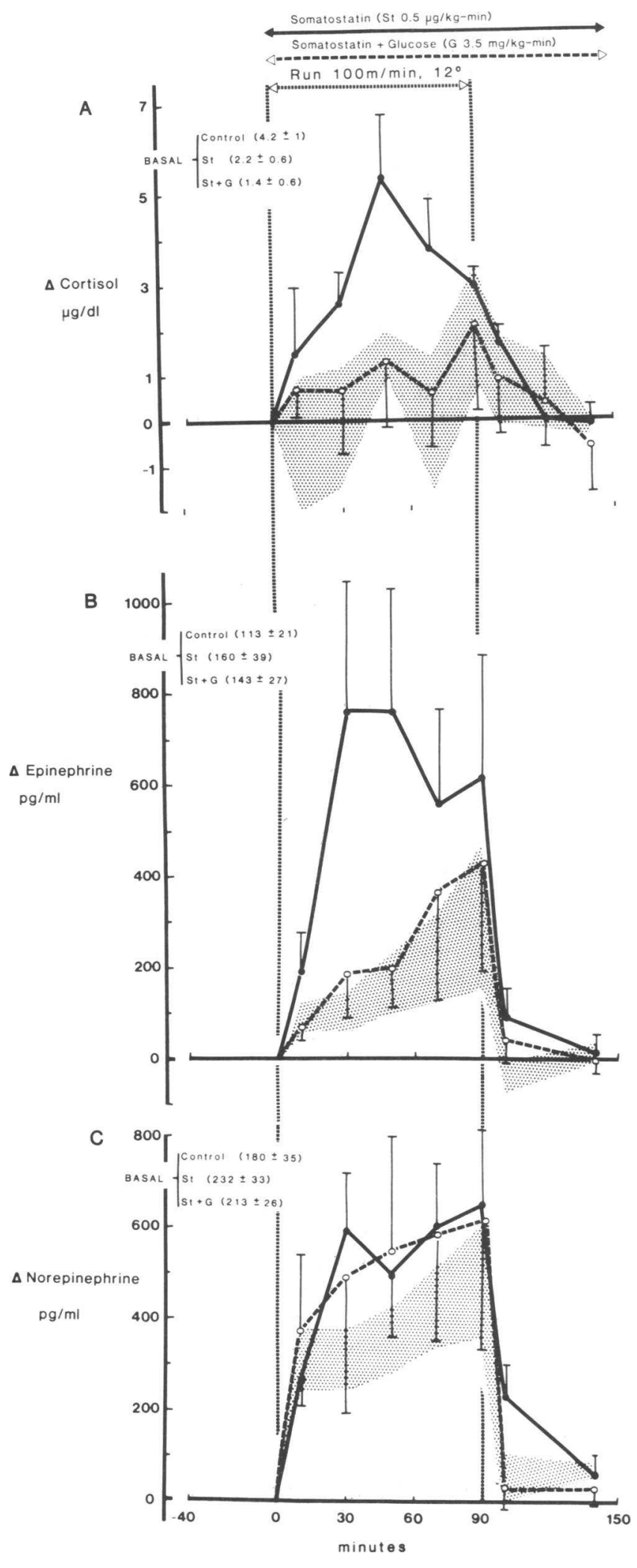

exercise in man and dog. In dog, glucagon increases gradually with the onset of exercise (in these studies glucagon doubled after $90 \mathrm{~min}$ ). In man, glucagon only increases in strenuous or prolonged exercise $(5,6)$.

With ST infusion during exercise, cortisol and epinephrine levels were both higher than during normoglycemic exercise (Fig. $5 A$ and $B$ ), whereas norepinephrine was not significantly different in any of the three protocols (Fig. $5 C$ ). These increases in epinephrine and cortisol occurred in response to the hypoglycemia that accompanied glucagon suppression, since these responses were alleviated when euglycemia was maintained during somatostatin infusion. The first significant change in epinephrine during exercise plus ST occurred at 10 min with a fall in blood glucose of only $8 \mathrm{mg} / \mathrm{dl}$. A similar decrease in glucose during rest does not lead to an increase in catecholamines (24-28). For example, in studies from our laboratory, epinephrine increased in normal resting dogs during insulininduced hypoglycemia only when plasma glucose fell $<60 \mathrm{mg} /$ $\mathrm{dl}(22,28)$. An increase in epinephrine has been observed with a moderate fall in plasma glucose (27), but this only occurred after $\mathbf{3 0} \mathrm{min}$ of continuous exposure to this degree of hypoglycemia. Thus, it appears that exercise can change the threshold for glucose, which generates a signal leading to an increased activity of the adrenal medulla. The fact that we observed a disproportionate increase in epinephrine but not in norepinephrine during hypoglycemia indicates that there may be separate control mechanisms for changes in adrenal medullary function and for changes in sympathetic drive. Cortisol also increases with moderate hypoglycemia in resting conscious dogs $(26,29)$ and this increase is similar to that observed during hypoglycemic exercise. Although the peak epinephrine value was attained at the same time as the nadir in plasma glucose, cortisol did not reach a peak until 20 min after this point. The time course of these responses could be consistent with a role for epinephrine in emergency situations and for cortisol in adaptation to stress. Recently, it has been suggested that the interplay between corticotropin and some of the brain neuropeptides can generate the selective hormonal responses in different stress situations (30).

To more clearly delineate the role of glucagon in dynamics of glucose production, we infused somatostatin with and without glucose replacement. In both groups receiving somatostatin, after an early nadir, glucagon rose at a rate similar to that seen in the controls, but still remaining well below the normal glucagon response to exercise. This transience is prob-

Figure 5. Effect of exercise alone (control, dotted area), exercise plus somatostatin (ST, $\longrightarrow$ ), or exercise plus somatostatin plus glucose $(\mathrm{ST}+\mathrm{G},----)$ on $(A)$ changes in cortisol concentrations, $(B)$ changes in epinephrine concentration, and $(C)$ changes in norepinephrine concentration. Data are shown as deviations $(\Delta)$ from the basal values. Exercise and somatostatin and glucose infusions were begun at $t=0$. The stippled area represents mean \pm SE for exercise alone. Vertical bars represent the SEM. $n=5$ for all protocols. 


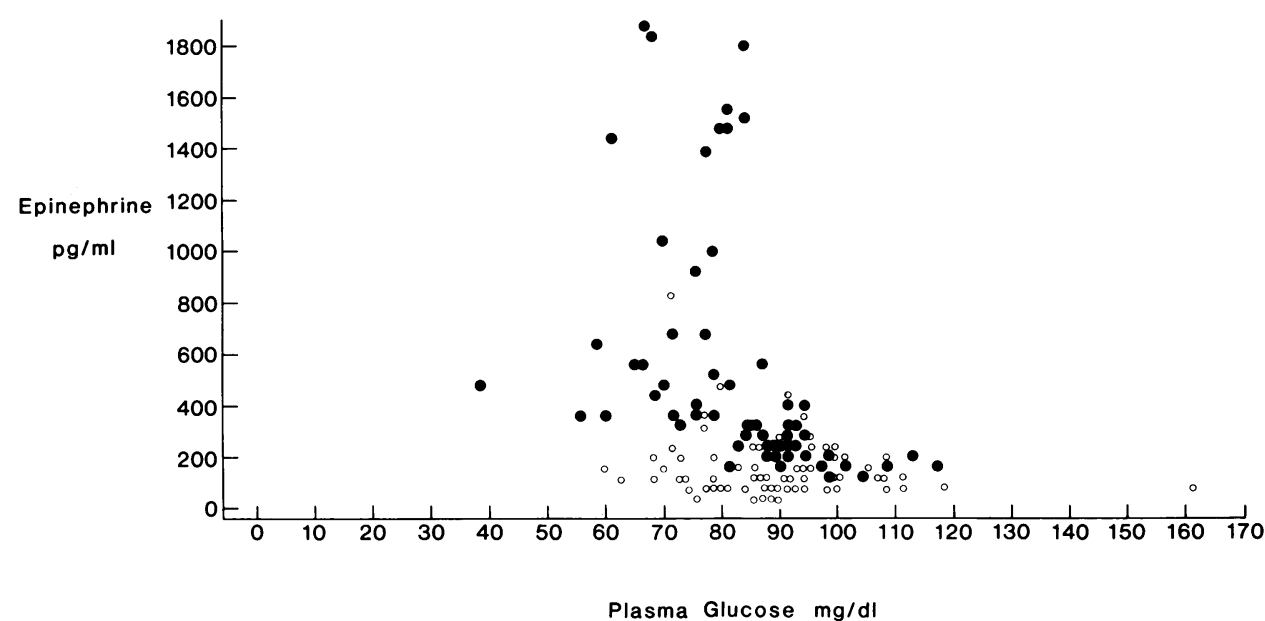

Figure 6. The relationship of plasma epinephrine levels to plasma glucose concentrations at rest (o) and during exercise (๑). ably a result of the characteristic adrenergic stimulation of glucagon seen with exercise (31). Glucose replacement abolished the compensatory hormonal responses seen when the dogs were allowed to become hypoglycemic during exercise, thereby isolating the effects of glucagon suppression from those of other counterregulatory hormones. The level of glycemia per se may affect $R_{a}$ directly $(32,33)$ or indirectly through altered secretion of pancreatic hormones $(34,35)$, which could in turn affect $R_{a}$. In this study, as in a previous study (9), there was an increase in $R_{a}$ with $S T$ infusion during exercise but this was much smaller than with exercise alone. This suppression was due to a delayed onset of the increase in $R_{a}$. With the infusion of ST plus $G$ during exercise, endogenous $R_{a}$ was suppressed an additional $2.0 \mathrm{mg} / \mathrm{kg}$-min with a similar time course (Fig. $2 \mathrm{~B}$ ). Thus, the full impact of glucagon suppression on $\mathbf{R}_{\mathbf{a}}$ is only revealed when the counterregulatory responses are prevented, as was also shown in resting dogs (10). It appears that during exercise, glucagon (to the extent it was suppressed in our study) in the presence of hypoinsulinemia is responsible for $75 \%$ of the $R_{a}$ increment seen at $90 \mathrm{~min}$. The maximal contribution of catecholamines could be $25 \%$ of the total $\mathbf{R}_{\mathbf{a}}$ response. During exercise with moderate hypoglycemia the contribution of glucagon is reduced to $60 \%$ of the endogenous $R_{a}$. This could be due both to an excessive release of epinephrine and to a direct effect of hypoglycemia on the liver. There is an interesting discrepancy between the responses of $\mathbf{R}_{\mathbf{a}}$ to changes in glucagon during exercise and in the resting state. Resting alloxan-diabetic dogs have IRI levels similar to those in exercising normal dogs. When epinephrine and glucagon were raised in these dogs to levels similar or exceeding those observed in exercising normal dogs, $R_{a}$ was initially increased as in exercise but only part of this increase was sustained (21). This difference can, in part, be explained because glucagon increases progressively during exercise while constant glucagon levels were maintained in the resting alloxandiabetic dogs. It is conceivable, however, that the lack of hyperglycemia and perhaps other unknown factors also con- tribute to the sustained effect of the glycogenolytic hormones during exercise.

Correlating $\mathbf{R}_{\mathbf{a}}$ with its potential hormonal regulators revealed a strong and highly significant positive correlation with the IRG/IRI molar ratio in all three experimental protocols (Fig. 4). This correlation was observed whether the results of the three experimental protocols in the present study were pooled or were considered independently. Although IRG alone was significantly correlated to $\mathbf{R}_{\mathbf{a}}$ when the data of the three protocols were pooled, independent analysis of each protocol showed a significant correlation only in control experiments. On the other hand, there was no significant correlation between $R_{a}$ and the catecholamines. Thus, it appears that IRG/IRI is the strongest determinant of $R_{a}$ in exercise.

With exercise plus ST, FFA's were significantly elevated when compared with values for exercise alone (Fig. 7 B) possibly due to excessive epinephrine release $(36,37)$. Functionally, a rise in FFA's with a falling blood glucose could provide the contracting muscle with an alternate source of fuel. Thus, the elevated FFAs may have a carbohydrate sparing effect. Surprisingly, however, the FFA levels were also elevated in the glucose clamp experiments despite normalized epinephrine values. The reason for these elevated FFA levels is not clear. It is possible that somatostatin can potentiate the effects of epinephrine on adipose tissue, since in resting dogs, combined infusion of ST and epinephrine also led to excessive FFA release (36). Although not significantly, the mean catecholamine level was slightly higher during the combined infusion of glucose and ST than in the control studies. If lipolysis during exercise is extremely sensitive to small changes in the catecholamines this may, at least in part, also explain the elevated FFA's in this group.

Plasma lactate was increased to a greater extent during hypoglycemic than during normoglycemic exercise (Fig. $7 A$ ). Since the increase in lactate occurred while glucose utilization was attenuated during hypoglycemia, the elevated lactate levels most probably reflect an increase in muscle glycogenolysis. 


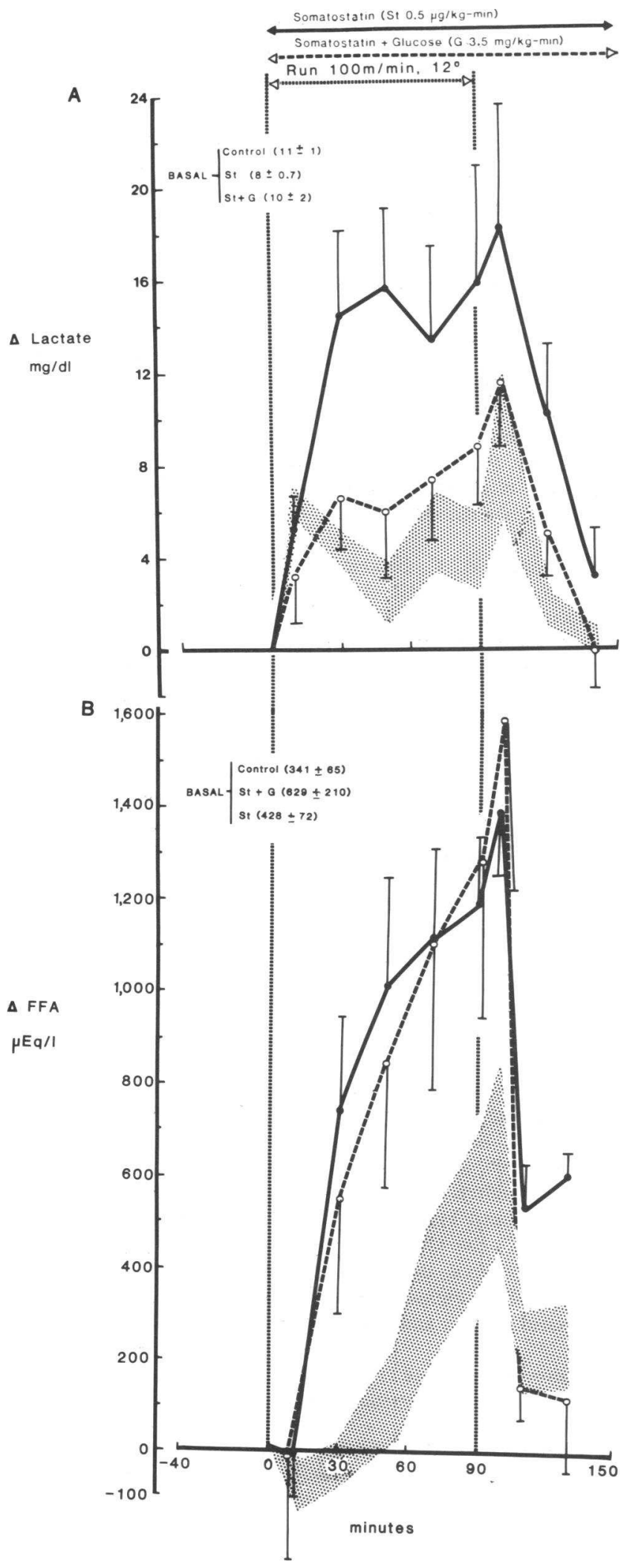

Theoretically, plasma lactate could also increase if lactate utilization in the liver is decreased by suppression of gluconeogenesis. This is unlikely, however, because changes in IRG and IRI that affected gluconeogenesis in resting dogs did not alter plasma lactate concentrations (20). In our experiments, endogenous $R_{a}$ was lowest with glucose replacement during exercise. Nevertheless, lactate levels were not increased but were the same as in control experiments. Thus, it appears that the increased plasma lactate seen during hypoglycemic exercise reflects an increased production rate. This could well relate to excessive epinephrine secretion since epinephrine increases lactate release from rat hindlimb (38) and lactate content in human muscle (39).

Compared with normoglycemic exercise, hypoglycemic exercise resulted in a decrease in glucose uptake (Fig. 2). This decrement was excessive compared with the fall in glucose and occurred despite similar insulin levels in all groups. The decrease in glucose uptake could reflect increased glycogenolysis and increased uptake of FFAs by muscle as a result of excessive epinephrine release (9). Beta adrenergic blockade decreases glycogenolysis in exercising dogs (37). Also, epinephrine increases the concentration of glycolytic intermediates in rat and human muscle $(38,39)$, of which glucose 6-phosphate is known to inhibit glucose uptake (40). With respect to FFA uptake, it has been shown that intermediates of fat metabolism can also suppress glucose uptake (41). Furthermore, recent work has shown that isoproterenol, a sympathomimetic agent, inhibits the recruitment of glucose transporters (42). Thus, when $\mathbf{R}_{\mathbf{a}}$ is decreased, a concurrent, relative suppression of glucose uptake provides another safeguard against hypoglycemia during exercise.

In conclusion, application of a glucose clamp during glucagon suppression in exercising dogs provides a model for delineating the role of glucagon during exercise. When glucagon suppression is accompanied by hypoglycemia there is an excessive release of epinephrine that has a moderate stimulatory effect on the liver and indirectly prevents a further fall in glucose by inhibiting glucose uptake by the muscle. With a euglycemic glucose clamp, the excessive counterregulatory response of epinephrine and cortisol are obviated and the lactate response to exercise is normalized. Comparison of normoglycemic and hypoglycemic exercise during glucagon suppression led us to hypothesize that when hepatic glucose production is decreased, glycogenolysis in the muscle is increased as a compensatory mechanism.

Figure 7. Effect of exercise alone (control, dotted area), exercise plus somatostatin (ST, - ), or exercise plus somatostatin plus glucose $\left(\mathrm{ST}+\mathrm{G},-{ }_{---)}\right.$on $(A)$ changes in lactate concentration, $(B)$ Changes in FFA concentration. Data are shown as deviations $(\Delta)$ from the basal values. Exercise and somatostatin and glucose infusions were begun at $t=0$. The stippled area represents mean $\pm \mathrm{SE}$ for exercise alone. Vertical bars represent the SEM. $n=5$ for all protocols. 


\section{Acknowledgments}

We are indebted to D. Bilinski, L. Cook, and N. Kovacevic for their excellent technical assistance. We are grateful to Dr. E. L. Lien (Wyeth Laboratories, Philadelphia, PA) for the generous gift of somatostatin. We would like to thank T. B. Jacobs for assistance in the data analysis and K. M. A. El Tayeb for valuable discussion during the course of these experiments.

This work was supported by the Medical Research Council of Canada, the Canadian Diabetes Association, Women's College Hospital Research Fund, and Bayer Pharmacological Research Centre (Wuppestal, Federal Republic of Germany). D. H. Wasserman is a recipient of an Edward Christie Stevens Fellowship of the University of Toronto.

\section{References}

1. Wahren, J., P. Felig, G. Ahlborg, and L. Jorfeldt. 1971. Glucose metabolism during leg exercise in man. J. Clin. Invest. 50:2715-2725.

2. Vranic, M., R. Kawamori, S. Pek, N. Kovacevic, and G. A. Wrenshall. 1976. The essentiality of insulin and the role of glucagon in regulating glucose utilization and production during strenuous exercise in dogs. J. Clin. Invest. 57:245-255.

3. Issekutz, B., Jr., A. C. Issekutz, and D. Nash. 1970. Mobilization of energy sources in exercising dogs. J. Appl. Physiol. 29:691-697.

4. Haggendahl, J., L. H. Hartley, and B. Saltin. 1970. Arterial noradrenaline concentration during exercise in relation to the relative work levels. Scand. J. Clin. Lab. Invest. 26:337-342.

5. Bottger, I., E. M. Schlein, G. R. Faloona, J. P. Knochel, and R. H. Unger. 1972. The effect of exercise on glucagon secretion. $J$. Clin. Endocrinol. Metab. 35:117-125.

6. Galbo, H., J. J. Holst, N. J. Christensen, and J. Hilsted. 1976. Glucagon and plasma catecholamines during beta-receptor in exercising man. J. Appl. Physiol. 40:855-863.

7. Galbo, H., N. J. Christensen, and J. J. Holst. 1977. Catecholamines and pancreatic hormones during autonomic blockade in exercising man. Acta Physiol. Scand. 101:428-437.

8. Hetenyi, G., G. Perez, and M. Vranic. 1983. Turnover and precursor-product relationships of nonlipid metabolites. Physiol. Rev. 63(2):606-667.

9. Issekutz, B., Jr., and M. Vranic. 1980. Significance of glucagon in the control of glucose production during exercise in dogs. Am. J. Physiol. 238(Endocrinol. Metab. 1): E13-E20.

10. Cherrington, A. D., J. E. Liljenquist, G. I. Shulman, P. E. Williams, and W. W. Lacy. 1979. Importance of hypoglycemia-induced glucose production during isolated glucagon deficiency. Am. J. Physiol. 236(Endocrinol. Metab. 3):E263-E271.

11. Steele, R. 1955. Influence of glucose loading and of injected insulin on hepatic glucose output. Ann. NY Acad. Sci. 82:420-430.

12. Cowan, J. S., and G. Hetenyi. 1971. Glucoregulatory responses in normal and diabetic dogs recorded by a new tracer method. Metab. Clin. Exp. 20:360-372.

13. Radziuk, J., K. H. Norwich, and M. Vranic. 1978. Experimental validation of measurements of glucose turnover in nonsteady state. Am. J. Physiol. 234(Endocrinol. Metab. Gastrointest. Physiol. 3):E84E93.

14. Herbert, V., K. S. Lau, C. W. Gottlieb, and S. Bleicher. 1965 Coated charcoal immunoassay of insulin. J. Clin. Endocrinol. Metab. 25:1375-1384.

15. Murphy, B. E. P. 1967. Some studies of the protein binding of steroids and their application to the routine micro and ultramicro measurement of various steroids in body fluids by competitive proteinbinding radioassay. J. Clin. Endocrinol. Metab. 27:973-990.

16. Hohorst, H. J. 1962. Lactate assay. In Methods of Enzymatic Analysis. H. U. Bermeyer, editor, Academic Press, Inc., New York. 266-270.

17. Faloona, G. R., and R. H. Unger. 1974. Glucagon. In Methods of Hormone Radioimmunoassays. B. M. Jaffe and H. R. Berman, editors, Academic Press, Inc., New York. 317-330.

18. Ho, R. J. 1970. Radiochemical assay of long-chain fatty acids using ${ }^{63} \mathrm{Ni}$ as tracer. Anal. Biochem. 36:105-113.

19. Sole, M. J., and M. N. Hussein. 1977. A simple specific radioenzymatic assay for the simultaneous measurement of picogram quantities of norepinephrine, epinephrine, and dopamine in plasma tissues. Biochem. Med. 18:301-307.

20. Cherrington, A. D., W. W. Lacy, and J.-L. Chiasson. 1978. The effects of glucagon on glucose production during insulin deficiency in the conscious dog. J. Clin. Invest. 62:664-677.

21. Perez, G., F. W. Kemmer, H. L. A. Lickley, M. Vranic. 1981. Importance of glucagon in mediating epinephrine-induced hyperglycemia in alloxan-diabetic dogs. Am. J. Physiol. 241(Endocrinol. Metab. 4):E328-E335.

22. Vranic, M., C. Gauthier, D. Bilinski, D. Wasserman, E. El Tayeb, G. Hetenyi, and L. Lickley. 1984. Catecholamine responses and their interactions with other glucoregulatory hormones. Am. J. Physiol. (Endocrinol. Metab.). In press.

23. Bjorkman, O., P. Felig, L. Hagenfeldt, and J. Wahren. 1981. Influence of hypoglucagonemia on splanchnic glucose output during leg exercise in man. Clin. Physiol. 1:43-57.

24. Christensen, N. J., K. G. M. M. Alberti, and O. Brandsborg. 1975. Plasma catecholamines and blood substrate concentrations: studies in insulin-induced hypoglycemia and after adrenaline infusions. Eur. J. Clin. Invest. 5:415-423.

25. DeFronzo, R. A., R. Andres, T. A. Bledsoe, G. Boden, G. Faloona, and J. A. Tobin. 1977. A test for the hypothesis that the rate of fall in glucose concentration triggers counterregulatory hormonal responses in man. Diabetes. 26:445-452.

26. Gauthier, C., M. Vranic, and G. Hetenyi. 1983. Nonhypoglycemic glucoregulation role of glycerol and glucoregulatory hormones. Am. J. Physiol. 244(Endocrinol. Metab. 7):E373-E379.

27. Santiago, J. V., W. L. Clarke, S. D. Shah, and P. E. Cryer. 1980. Epinephrine, norepinephrine, glucagon, and growth hormone release in association with physiological decrements in the plasma glucose concentration in normal and diabetic man. J. Clin. Endocrinol. Metab. 51:872-883.

28. El Tayeb, K. M. A., P. L. Brubaker, H. L. A. Lickley, and M. Vranic. 1984. Effects of naloxone on hormonal and metabolic responses to insulin-induced hypoglycemia. 7th International Congress of Endocrinology, Quebec City, July 1984. 434. (Abstr.)

29. Keller-Wood, M. E., J. Shinsako, L. C. Kiel, and M. F. Dallman. 1981. Insulin-induced hypoglycemia in conscious dogs. I. Dose related pituitary and adrenal responses. Endocrinology. 109:818824.

30. Brown, M. R., and L. A. Fisher. 1984. Brain peptide regulation of adrenal epinephrine secretion. Am. J. Physiol. (Endocrinol. Metab. 10). 247:E41-E46.

31. Luyckx, A. S., F. Pirnay, A. Cession-Fossion, and P. J. Lefebvre. 1975. Catecholamines and exercise-induced glucagon and fatty acid mobilization in the rat. Am. J. Physiol. 229:376-383.

32. Bergman, R. N. 1977. Integrated control of hepatic glucose metabolism. Fed. Proc. 36:265-270. 
33. Sacca, L., R. Hendler, and R. S. Sherwin. 1979. Hyperglycemia inhibits glucose production in man independent of changes in glucoregulatory hormones. J. Clin. Endocrinol. Metab. 47:1160-1163.

34. Iversen, J. 1971. Secretion of glucagon from the isolated perfused canine pancreas. J. Clin. Invest. 50:2123-2136.

35. Ohneda, A., E. Aguillar-Parada, A. M. Eisentraut, and R. H. Unger. 1969. Control of pancreatic glucagon secretion by glucose. Diabetes. 18:1-10.

36. Gray, D. E., H. L. A. Lickley, and M. Vranic. 1980. Physiological effects of epinephrine on glucose turnover and plasma free fatty acid concentration mediated independently of glucagon. Diabetes. 29:600 609.

37. Issekutz, B. 1978. Role of beta-adrenergic receptors in mobilization of energy sources in exercising dogs. J. Appl. Physiol. Respir. Environ. Exercise Physiol. 44:869-876.

38. Richter, E. A., N. B. Ruderman, H. Gavras, E. R. Belur, and
H. Galbo. 1982. Muscle glycogenolysis during exercise: dual control by epinephrine and contractions. Am. J. Physiol. 242(Endocrinol. Metab. 5):E25-E32.

39. Chasiostis, D., K. Sahlin, and E. Hultman. 1983. Regulation of glycogenolysis in human muscle in response to epinephrine infusion. J. Appl. Physiol. Respir. Environ. Exercise Physiol. 54:45-50.

40. Colowick, S. P. 1973. The hexokinases. In The Enzymes. P. D. Boyer, editor. Academic Press, Inc., New York. Third ed. 9:1-48.

41. Randle, P. J., and G. H. Smith. 1958. Regulation of glucose uptake by muscle. II. The effect of insulin, anaerobiosis and cell poisons on the penetration of isolated rat diaphragm by sugars. Biochem. J. 70:502-508.

42. Simpson, I. A., U. Smith, and S. W. Cushman. 1983. Counterregulation of insulin-stimulated glucose transport by isoproterenol in rat adipose cells: modulation of intrinsic glucose transporter activity. Diabetes. 32(Supp.):33a. (Abstr.) 